\title{
Identifying VOCs in exhibition cases and efflorescence on museum objects exhibited at Smithsonian's National Museum of the American Indian-New York
}

\author{
Alba Alvarez-Martin 1,5*0 ${ }^{10}$, John George ${ }^{2}$, Emily Kaplan ${ }^{3}$, Lauren Osmond ${ }^{3}$, Leah Bright ${ }^{3}$, G. Asher Newsome ${ }^{1}$, \\ Rebecca Kaczkowski ${ }^{1}$, Frederik Vanmeert ${ }^{4}$, Gwénaëlle Kavich ${ }^{1}$ and Susan Heald ${ }^{3}$
}

\begin{abstract}
Two mass spectrometry (MS) methods, solid-phase microextraction gas chromatography (SPME-GC-MS) and direct analysis in real time (DART-MS), have been explored to investigate widespread efflorescence observed on exhibited objects at the Smithsonian's National Museum of the American Indian in New York (NMAI-NY). Both methods show great potential, in terms of speed of analysis and level of information, for identifying the organic component of the efflorescence as 2,2,6,6-tetramethyl-4-piperidinol (TMP-ol) emitted by the structural adhesive (Terostat MS 937) used for exhibit case construction. The utility of DART-MS was proven by detecting the presence of TMP-ol in construction materials in a fraction of the time and effort required for SPME-GC-MS analysis. In parallel, an unobtrusive SPME sampling strategy was used to detect volatile organic compounds (VOCs) accumulated in the exhibition cases. This sampling technique can be performed by collections and conservation staff at the museum and shipped to an offsite laboratory for analysis. This broadens the accessibility of MS techniques to museums without access to instrumentation or in-house analysis capabilities.
\end{abstract}

Keywords: Solid phase microextraction-gas chromatography, Direct analysis in real time, Volatile organic compounds, Material analysis, Efflorescence, Preventive conservation

\section{Introduction}

In 2015, five years after opening the exhibition Infinity of Nations: Art and History in the Collection of the National Museum of the American Indian at the Smithsonian's National Museum of the American Indian in New York (NMAI-NY), staff observed a white crystalline efflorescence on objects in display cases manufactured for the exhibit (Fig. 1).

Other museums have described similar issues, observing efflorescence in exhibition cases from the same

\footnotetext{
*Correspondence: alba.alvarez@usal.es

5 Present Address: Conservation \& Science, Rijksmuseum, Hobbemastraat 22, 1071 ZC Amsterdam, The Netherlands

Full list of author information is available at the end of the article
}

manufacturer installed between 2009 and 2014 [1-3]. While other museums have noted efflorescence on case sealing gaskets, glass door interiors, and occasionally on certain types of objects, at NMAI-NY the efflorescence is not observed on gasket materials but appears on a variety of component materials of collections items in these exhibit cases: ceramic, stone, wood, plant fibers and seeds, feathers, fur, textiles, and tanned hide. While the efflorescence is found mostly on archaeological materials, it is also found on historic and contemporary objects. This was documented in an object-by-object survey conducted in 2016 which concluded that about one quarter of the collection items displayed in Infinity of Nations had developed efflorescence. During a pilot cleaning campaign on 15 artifacts in 2017, conservators described
Springer Open

(c) The Author(s) 2020. This article is licensed under a Creative Commons Attribution 4.0 International License, which permits use, sharing adaptation, distribution and reproduction in any medium or format, as long as you give appropriate credit to the original author(s) and the source, provide a link to the Creative Commons licence, and indicate if changes were made. The images or other third party material in this article are included in the article's Creative Commons licence, unless indicated otherwise in a credit line to the material. If material is not included in the article's Creative Commons licence and your intended use is not permitted by statutory regulation or exceeds the permitted use, you will need to obtain permission directly from the copyright holder. To view a copy of this licence, visit http://creativeco mmons.org/licenses/by/4.0/. The Creative Commons Public Domain Dedication waiver (http://creativecommons.org/publicdomain/ zero/1.0/) applies to the data made available in this article, unless otherwise stated in a credit line to the data. 


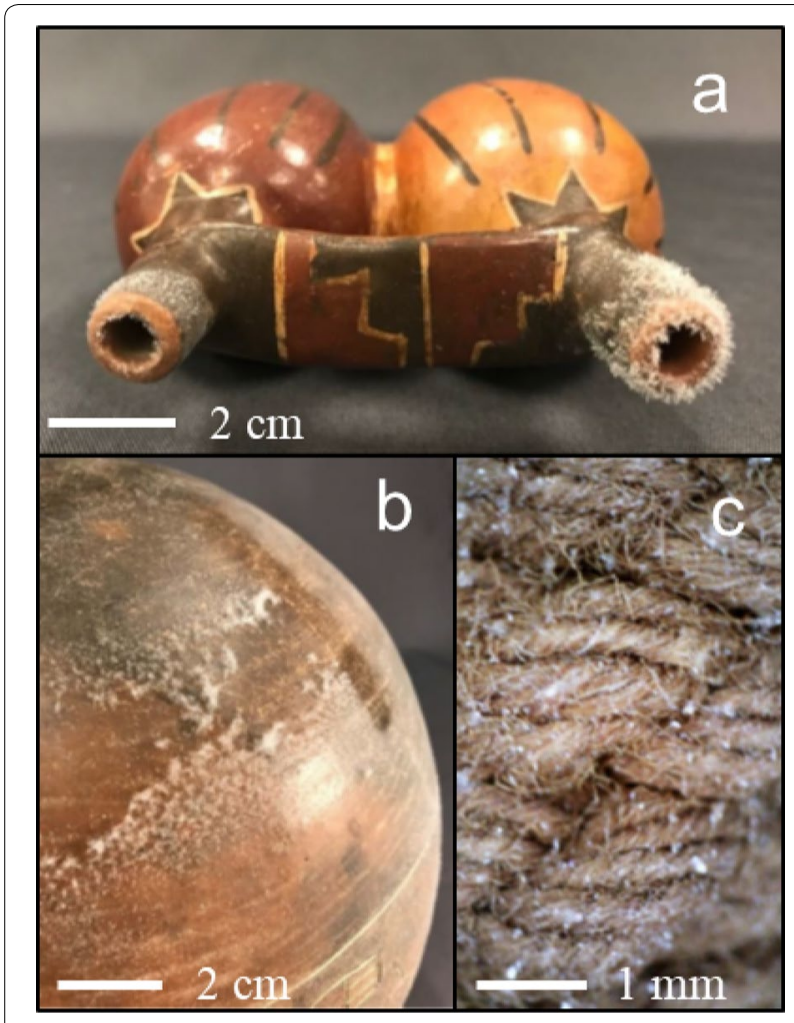

Fig. 1 Photographs of efflorescence on a a Nazca double spout ceramic vessel; 17/8902, b Paracas ceramic vessel; 23/5500, and c micrograph of efflorescence formed on cotton cordage knot of Inka quipu; 14/3866

different types of efflorescence depending on the nature of the object: 'heavy crystal growth' was found in archaeological ceramics and several organic materials. Painted surfaces presented 'sparkling frost-like' efflorescence. Though most crystals appeared 'light and feathery', they were described as 'waxy and compacted' when touched, causing suspicion that the substance might remain on the surfaces even after cleaning. Unfortunately, there is no related literature on the effect this type of efflorescence has on museum collections and no successful cleaning strategies have been published.

The first study focused on this issue, carried out by the Museum of Fine Arts, Boston (USA) and the University of Turin (Italy), suggested that the formation of this nonvolatile substance may have been caused by a reaction between the volatile 2,2,6,6-tetramethyl-4-piperidinol (TMP-ol) and an acidic compound, containing chlorine or sodium, also present in the case environment $[1,2]$. In a recent publication by the Rijksmuseum, five types of crystalline deposits are described as a result of an acidbasic reaction between TMP-ol and different carboxylic acids (2,4-dichlorobenzoic acid, formic acid, methacrylic acid, palmitic acid and an unknown carboxylic acid) emitted from peroxide-cured silicone gaskets, panels of medium-density fibreboard (MDF), UV adhesive, beeswax containing products, and from an unknown acidic conservation product or binding medium [3].

TMP-ol is a component of hindered amine light stabilizers (HALS), such as Uvasorb HA 88, Cyasorb UV-3529, and Tinuvin ${ }^{\circledR} 770$, widely used as additives during the manufacture of polymers to prevent thermooxidative and light-induced degradation. Tinuvin ${ }^{\circledR} 770$ is an additive of the structural adhesive (Terostat) applied by the casework manufacturer from 2009 through 2014 . Tinuvin ${ }^{\circledR} 770$ or bis $(2,2,6,6$,-tetramethyl-4-piperidyl) sebaceate is synthetized by the reaction of TMP-ol with an appropriate sebacic acid derivative such as dimethyl sebacate [4] or sebacic acid [5]. According to the case manufacturer, two different formulations of the structural adhesive were used during the installation at NMAI-NYC: Terostat MS 937 (white and grey) and Terostat 9220 (black). However, to date, only Terostat 937 adhesive has been found in the cases at NMAI. Terostat is classified as modified silane polymer sealant with good capacity to bond various substrates together such as stainless steel, aluminum and other metals, glass, plastics, wood, and painted surfaces.

Although the possible interaction between Tinuvin ${ }^{\circledR}$ 770 and other analytes such as acids has been documented [6], museum staff did not anticipate problems with the adhesive because it had been vetted via Oddy testing [7]. Although an extended length of time is required to obtain results, the Oddy test is still widely used in cultural institutions as a preliminary means to evaluate potentially reactive materials. The test has been criticized because the results are not representative of potential damage for the wide variety of materials represented in museum collections. In addition, as materials are tested individually, the test cannot provide information on reactions between materials $[8,9]$. Furthermore, evaluating the results of the Oddy test is subjective and cannot identify specific volatile organic compounds (VOCs) released by test samples. Since this test is insufficient to conclusively identify the long-term compatibility of materials, other strategies for material testing are being evaluated in cultural institutions. In order to fulfil this objective, several mass spectrometry (MS) approaches have been explored as faster and more comprehensive methods to detect off-gassing VOCs from museum construction materials [10-13]. However, most studies have focused on laboratory experiments, which require a sample to be taken from the material (invasive analysis). For on-site detection of VOCs inside showcases the sampling setup of air sampling devices or sensors can be visually disruptive to the exhibition. Thanks to the miniaturization of traditional sample preparation devices for MS this 
technique can become more suitable for the analysis of historic artifacts.

Solid-phase microextraction (SPME) was developed by Pawliszyn in 1989 [14] for rapid analysis in the laboratory and at a remote site. The sampling is performed using a fiber, housed in a stainless-steel needle, coated with different sorbent phases capable of extracting a wide range of analytes, from volatile to non-volatile and from polar to non-polar. After sampling the analytes are thermally desorbed and transferred to a mass spectrometer. The easy and compact SPME sampling approach presents a significant advantage over conventional sampling methods (e.g. Tenax ${ }^{\circledR}$ tubes, Drager ${ }^{\circledR}$ tubes), that may use a larger assembly kit and may require a mounting system in the display case.

In addition, the interfacing of SPME with an ambient mass spectrometry technique, such as direct analysis in real time mass spectrometry (SPME-DART-MS), is a potential tool for evaluating specific VOCs that are hazardous to museum objects, such as small carboxylic acids [15]. DART uses a continuous heated flow of post-plasma gas to desorb and ionize analytes from the sample's surface at atmospheric pressure. By using the selectivity of high-resolution mass analysis or selected reaction monitoring to discern analytes, DART is operated without chromatographic separation. The small volume of the SPME coating makes it well-suited to interface with DART [16] by using the high-temperature gas to desorb analytes from the fiber. Although DART has been used for qualitative characterization of polymers and polymer additives [17], the application of DART-MS to investigate exhibition case materials has not previously been assessed.

The goal of this project was to optimize and evaluate two rapid and high sensitivity analytical mass spectrometry approaches, SPME-GC-MS and DART-MS, with the aim to:

1. Characterize the (organic-based) efflorescence and its volatile components;

2. Develop rapid screening of construction materials in order to identify the possible sources of the volatile components deposited as efflorescence;

3. Perform on-site sampling of the VOCs accumulated within the exhibition cases to predict the formation of future efflorescence;

4. Optimize a reproducible and 'user friendly' sampling protocol and transportation methodology.

\section{Description of the exhibition}

Infinity of Nations is composed of 33 display cases: 32 designed and manufactured by a contracted fabricator and one display case built in-house. Twelve are wall cases - ten with swinging door access and two with sliding doors; there are ten free-standing cases, nine semi-circular cases, and one horizontal hydraulic case distributed in $604 \mathrm{~m}^{2}$ of gallery space (Fig. 2).

Major construction materials are glass and metal. All materials including glass front, sides, and tops,

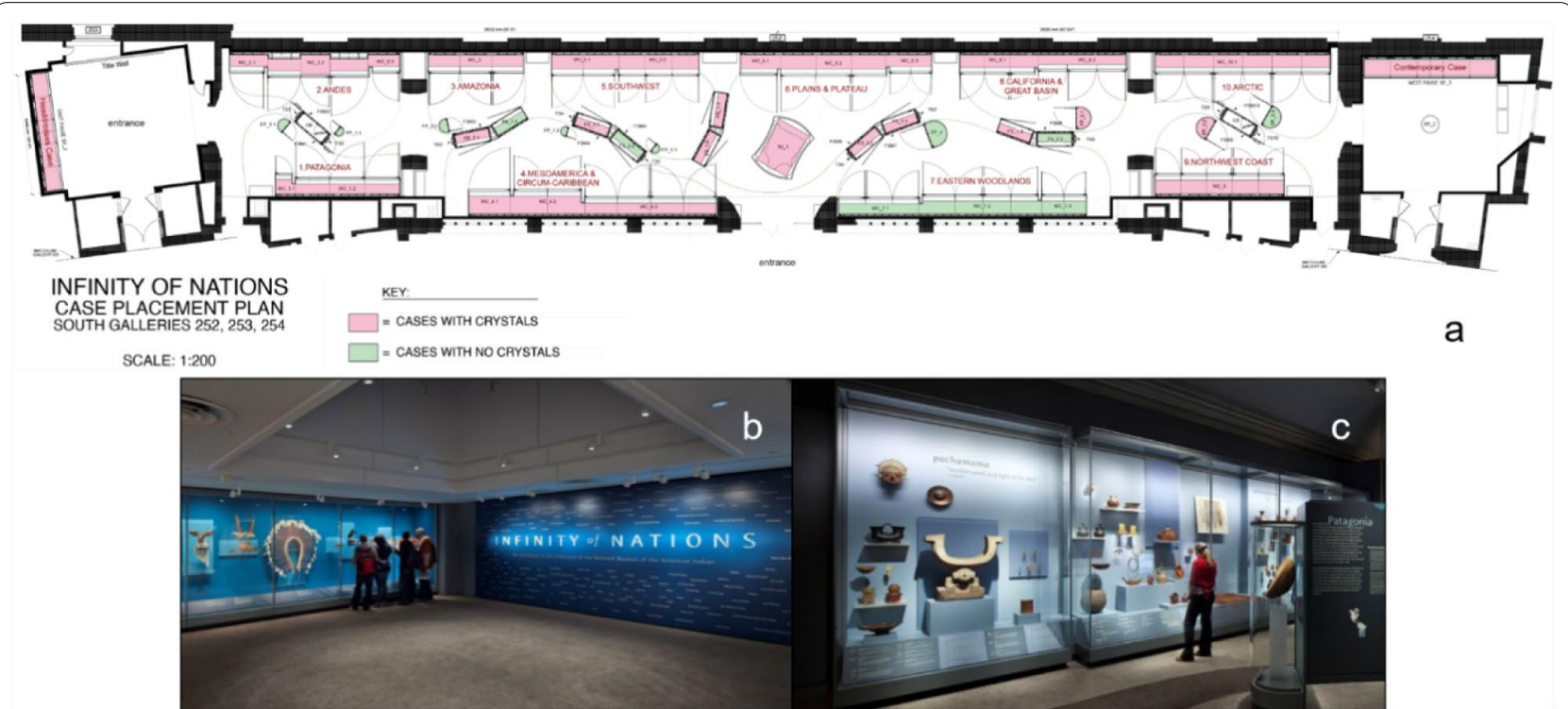

Fig. 2 a Infinity of Nations exhibition floor plan, the pink areas indicate cases with efflorescence on objects. Views of: $\mathbf{b}$ entrance of the exhibition and Headdresses wall case and $\mathbf{c}$ Andes wall case 
powder-coated steel, decks and case backs are Appleply, a birch plywood (made without urea formaldehyde adhesives) covered with a barrier of aluminum sign blank which is painted with a low VOC paint (see SI for a detailed description of the construction materials). The cases seal tightly and are designed with desiccant drawers and fans to maintain air circulation within the cases.

To date, efflorescence has been detected on a total of 163 out of 694 examined objects in 21 of the 32 display cases designed and manufactured by a contracted fabricator. No efflorescence has been detected in the display case built in-house.

\section{Materials}

A $75 \mu \mathrm{m}$ carboxen-poly(dimethylsiloxane) SPME fiber (CAR/PDMS, 57344-U Supelco) was purchased from Sigma-Aldrich (USA).

PTFE/silicone septa (9301-0719), $10 \mathrm{~mL}$ headspace sample vials (5182-0838), and $20 \mathrm{~mm}$ aluminum crimp caps (9301-0718) were purchased from Agilent (USA). Deactivated glass inlet liners for capillary injection port (2637505, Supelco) and Thermogreen ${ }^{\circledR}$ LB-2 pre-drilled septa (23268) were purchased from Sigma-Aldrich (USA).

\section{Experimental}

Efflorescence

During four sampling campaigns (December 2018, February 2019, March 2019, and February 2020), eleven efflorescence samples were collected from eight different objects (see Additional file 1: Table S1 for detailed description). The most effective methods for sampling crystals were by scraping, swabbing, or brushing the crystals onto a glass well-slide. Samples were transferred from the glass slide to glass vials and stored with no light exposure at room temperature until analysis was conducted.

\section{Construction materials}

Thanks to records compiled by museum staff during casework installation at NMAI-NY, it was possible to sample the different types of construction materials applied to specific components of each case type (Fig. 3). 26 samples of case construction materials were taken from the display cases, of which 18 were classified as structural adhesive (16 white samples and two grey samples, all Terostat MS 937), three as magnetic (door) gasket, three as silicone (desiccant drawer) gasket, one paint sample, and one silica gel sample from the desiccant drawer (see Additional file 1: Table S2).

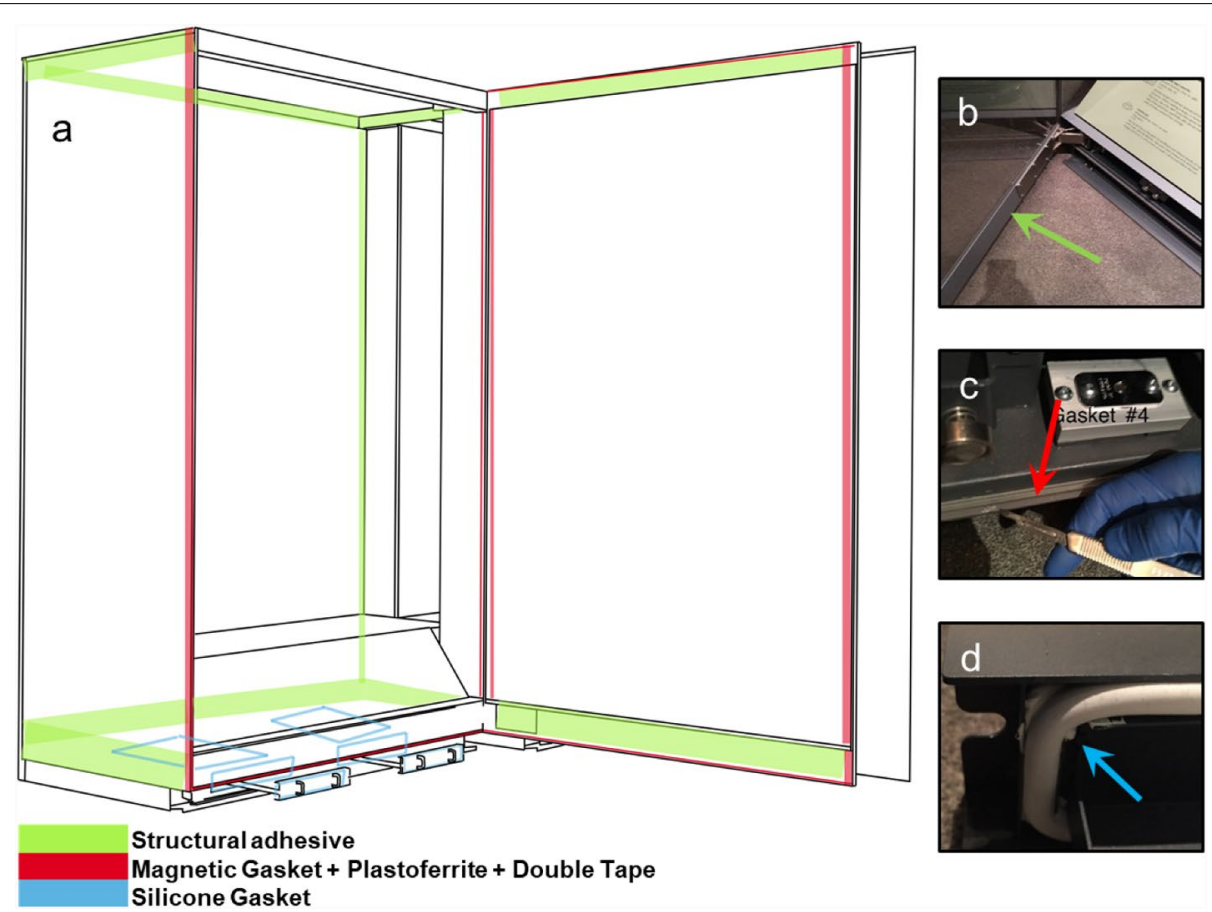

Fig. 3 a Infinity of Nations exhibition case design. Green areas indicate surfaces where the structural adhesive was applied, red areas indicate the location of the magnetic (door) gasket and blue areas indicate the location of the silicone (desiccant drawer) gasket. Sampling locations of $\mathbf{b}$ structural adhesive, c magnetic (door) gasket and $\mathbf{d}$ silicone (desiccant drawer) gasket are indicated with arrows 
Construction materials collected at NMAI-NY were also compared with samples collected from the Cooper Hewitt Smithsonian Design Museum (CHSDM, New York) and the Smithsonian National Museum of African American History and Culture (NMAAHC, Washington, DC) exhibition cases. These cases were produced by the same manufacturer and installed in 2014 and 2016, respectively. One sample of structural adhesive from the CHSDM and one structural adhesive and two magnetic gaskets from NMAAHC were sampled in March 2019.

\section{SPME sampling}

\section{Efflorescence and construction materials}

Both the efflorescence and construction material samples were individually sampled in $10 \mathrm{~mL}$ headspace vials, where the SPME fiber (CAR/PDMS) was exposed to the sample headspace for $24 \mathrm{~h}$ at room temperature (average of $21.5{ }^{\circ} \mathrm{C}$ ). The weight and dimension of each sample collected from the exhibition varied depending on its accessibility, and, therefore, the weight of each material was not considered.

\section{Display cases and exhibition hall: on-site sampling}

The main purpose of the on-site analysis was to characterize VOCs accumulated in exhibition cases with and without efflorescence to detect differences. 32 SPME fibers were exposed for $24 \mathrm{~h}$ in different locations during the four campaigns between 2018 and 2020 (Additional file 1: Table S3). When cases are opened, VOC concentration decreases. However, after $24 \mathrm{~h}$ the VOCs concentration is again sufficiently high to be detected by SPME-GC-MS $[18,19]$. The selection of the sampling points was based on the visual observation of crystals made by the museum conservators and collection manager in a first exploratory phase. In total, 11 exhibition cases were investigated; 8 exhibition cases where efflorescence was observed and three where efflorescence was not observed. The sampling strategy was tailored for each display case (Fig. 4a, b), where SPME fibers were placed in the headspace of the case without touching any of the objects.

To record the background VOCs of the exhibition gallery in contrast with the cases, SPME fibers were placed on top and below two exhibition cases (Additional file 1: Table S3).

\section{Direct contact analysis of objects: on-site sampling}

In order to enhance the detection of the efflorescence without physical sampling (e.g., removing crystals by scraping or swabbing), direct-contact SPME analysis was performed on four objects (Additional file 1: Table S3). For this the fiber was placed in contact with the surface of the object during the $24 \mathrm{~h}$ sampling period (Fig. $4 \mathrm{c}$ ).

\section{Transportation}

Two different modes of transporting the SPME fibers between NMAI-NY (New York, USA) and the Museum Conservation Institute (MCI) (Maryland, USA) were evaluated to assess whether possible fluctuations in environment and handling would affect the results. From the 32 SPME fibers exposed at NMAI-NY, one set of two fibers was shipped via express mail from the MCI analytical laboratories to the NMAI-NY museum (Additional file 1: Table S3). A second set of two fibers were handcarried via train from the MCI to the NMAI-NY. The pairs of fibers, one shipped and one hand-carried, were exposed in the same location for $24 \mathrm{~h}$ on-site sampling. After sampling, the fibers were sent back to the MCI by express mail and hand-carried. When removed from the showcase the fibers were sealed with a Thermogreen ${ }^{\circledR}$ LB-2 pre-drilled septa with an extremely low bleed (what is used to seal the GC injector port). All fibers were analyzed by GC-MS within $24 \mathrm{~h}$ after sampling.

\section{SPME-GC-MS}

After sampling, SPME fibers were manually inserted into the injector port of the GC with a Supelco SPME holder (Sigma Aldrich, 57330-U). VOCs were thermally desorbed at $270{ }^{\circ} \mathrm{C}$ from the fiber and transferred onto the chromatographic column for separation prior to detection via the mass spectrometer. GC-MS analyses were performed with an Agilent 6890 GC coupled to a 5975 MSD (Agilent Technologies, Wilmington, DE, USA). Chromatographic separation was performed on a $30 \mathrm{~m} \times 0.25 \mathrm{~mm}$ i.d., $0.25 \mu \mathrm{m}$-thick film of DB-5MS capillary column (Agilent Technologies, Wilmington, DE, USA). The injector was used in pulsed-splitless mode with a total injection time of $4 \mathrm{~min}$. The carrier gas was helium with a constant flow of $1 \mathrm{~mL} / \mathrm{min}$. The oven program was as follows: initial temperature of $40{ }^{\circ} \mathrm{C}$ (hold for $10 \mathrm{~min}$ ), ramp rate of $5{ }^{\circ} \mathrm{C} / \mathrm{min}$ to $270{ }^{\circ} \mathrm{C}$, which was held for $10 \mathrm{~min}$. The temperature of the interface and the source were set at $280{ }^{\circ} \mathrm{C}$ and $230{ }^{\circ} \mathrm{C}$, respectively. Mass spectra were acquired under electron ionization mode (EI) at $70 \mathrm{eV}$ and recorded from $\mathrm{m} / \mathrm{z}$ 40-300 with a scan time of $0.19 \mathrm{~s}$. Data analysis was performed using Masshunter ${ }^{\circledR}$ qualitative analysis software (version B.07.00, Agilent). Chromatographic peaks were identified using the NIST library 2011 Mass spectra Library V.2.0. The GC-MS data generated in this study were only used for qualitative interpretation.

\section{DART-MS}

A DART-100 probe using helium discharge gas and operated by a standardized voltage and pressure (SVP) controller (IonSense, Saugus, MA) was custom-mounted to interface to a Linear Trap Quadropole (LTQ) Orbitrap 

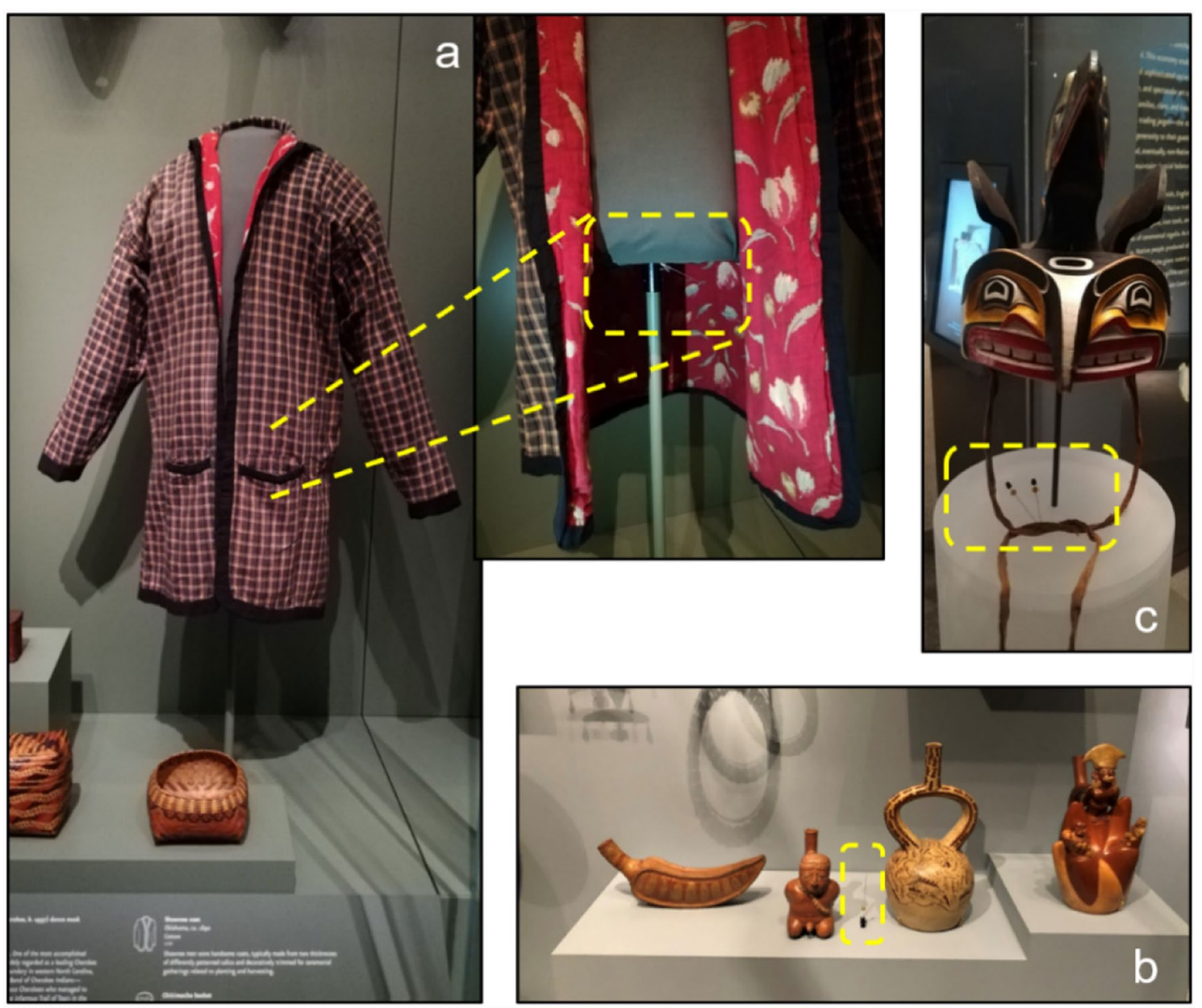

Fig. 4 On-site sampling locations: a Eastern Woodlands wall case, SPME fiber mounted into internal Ethafoam ${ }^{\circledR}$ support of Oklahoma Shawnee coat; NMAI 02/0528, b Andes wall case, SPME fiber in back of shelf between two Moche stirrup spout ceramic vessels; NMAI 23/8958 and NMAI 16/8980, c Northwest Coast focal point case, paired SPME fibers in direct contact with efflorescence on cotton ties of Kwakwaka'wakw headdress, wood, paint, cotton fabrics; NMAI 23/8252

Velos mass spectrometer (Thermo Fisher Scientific, Waltham, MA). The ceramic transfer tube in the flange was replaced with a $64 \mathrm{~mm}$ o.d., $40 \mathrm{~mm}$ long stainless steel tube [15].

A small fragment of each construction material sample was held in place between the DART ionization source and the MS inlet using tweezers (Additional file 1: Fig. S1a). For the analysis of the efflorescence, a small amount of material was sampled with the end of a glass tip and placed between the source and the inlet (Additional file 1: Fig. S1b).

For all analyses, the temperature of the helium plasma was set to $200{ }^{\circ} \mathrm{C}$. Mass spectra were acquired in positive mode from $\mathrm{m} / \mathrm{z} 50$ to 500 at a resolving power of 30,000. Data analysis was performed using Xcalibur Qual Browser V.3.0 (Thermo Fisher Scientific). No quantitative
DART-MS interpretation, e.g. using isotopically-labelled standards, was required for this study.

\section{Results and discussion}

\section{Characterization of the efflorescence}

During the exploratory stage, Scanning Electron Microscopy-Energy Dispersive X-ray Spectroscopy (SEM-EDS) analysis was used to investigate the elemental composition of the efflorescence (experimental details in SI and Additional file 1: Table S1, Figs. S2 and S3) and highlights the presence of chlorine $(\mathrm{Cl})$ reported by previous studies, where the efflorescence was described as a chlorinated salt $[1,2]$. Additional analyses by XRPD and ATR-FTIR were made to further identify the composition of the efflorescence and its crystalline material. However, no positive identification could be made from the available databases (experimental details and results 
are described in Additional file 1: Table S1, Figs. S2 and S3).

In order to better characterize the chemical composition of the efflorescence, SPME-GC-MS and DART-MS were applied in all efflorescence samples collected from the exhibition.

SPME-GC-MS analysis of the efflorescence samples presented a main peak at 20.2 min retention time (Fig. 5a) and confirmed the presence of TMP-ol (Fig. 5b, c). All efflorescence samples collected at NMAI-NY were also analyzed by DART-MS, showing the ion peak $(m / z 158.1527)$ corresponding to the protonated TMPol $\left(\left[\mathrm{C}_{9} \mathrm{H}_{19} \mathrm{NO}+\mathrm{H}\right]^{+}\right)$(Fig. $5 \mathrm{~d}$ ). No other peaks have been identified by both MS techniques, showing that the organic component of the efflorescence is a piperidinolbased compound (Table 1).

With the organic component of the efflorescence characterized, efforts were focused on determining the source of TMP-ol and the origin of chlorinated compounds that can form the chlorinated salt, reported in previous studies $[1,3]$ and detected in this study by SEM-EDS analysis.

\section{Source of the efflorescence: analysis of construction} materials

DART-MS analyses were performed on 26 samples identified as construction materials. A subset of 17 samples, comprising all types of sampled construction materials, were analyzed by SPME-GC-MS in order to obtain a comprehensive overview of the different VOCs emitted by these materials (Additional file 1: Table S2).

The main components released by both gasket materials and the structural adhesive were found to be siloxane oligomers. As no siloxane oligomers have been detected in the efflorescence, no significance was ascribed to these compounds.

TMP-ol, which is a constituent of the efflorescence, was detected in 9 of the 17 analyzed materials, all of them classified as structural adhesive (Terostat MS 937) (Table 1). Interestingly, dimethyl sebacate was also identified in the structural adhesive (Terostat MS 937), while Tinuvin 770 (Fig. 5e), a combination of two molecules of TMP-ol and one dimethyl sebacate, was not detected in any of these samples.
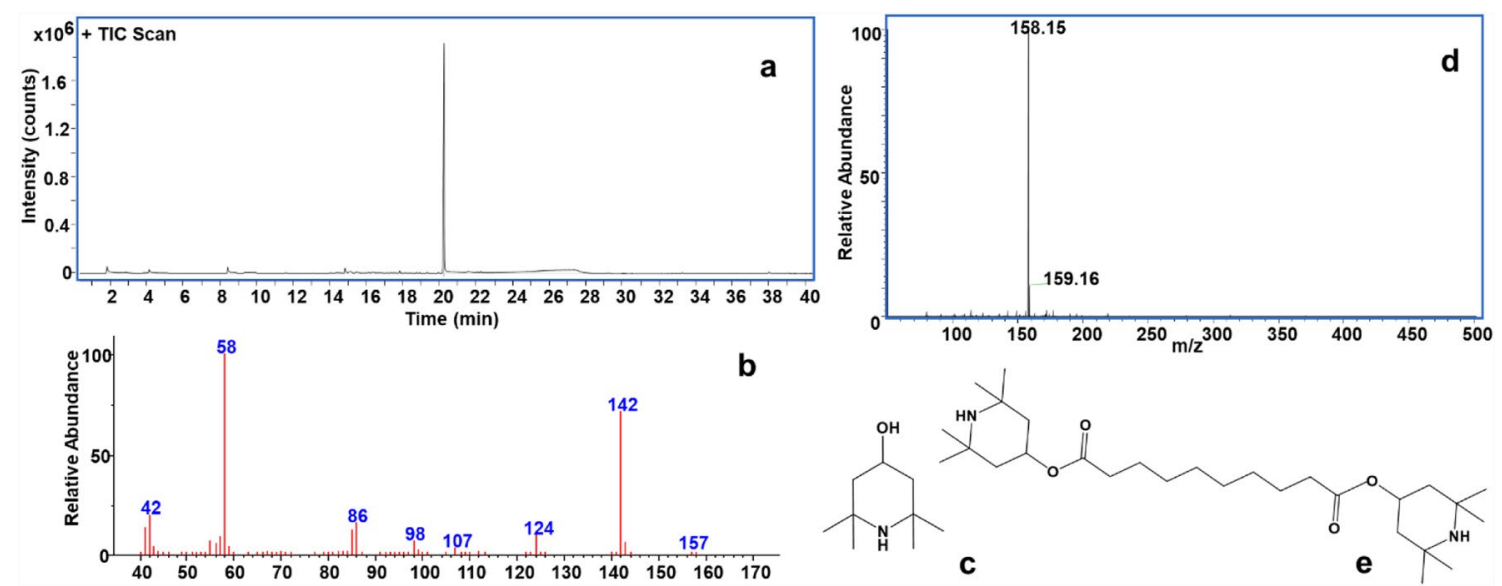

Fig. 5 a GC-MS Total lon Chromatogram (TIC) profile of efflorescence collected from Pueblo bowl (NMAI 12/8961) by SPME-GC-MS sampling. b GC-MS spectrum of the most abundant peak with retention time 20.242 min, with a $91 \%$ NIST library spectrum matching for 2,2,6,6-tetramethyl-4-p iperidinol (TMP-ol) (CAS No. 2403-88-5). c Chemical structure of 2,2,6,6-tetramethyl-4-piperidinol (TMP-ol) d DART-MS spectrum of the efflorescence collected from Pueblo bowl (NMAl 12/8961) with ion peak (m/z 158.1527, $\left.\left[\mathrm{C}_{9} \mathrm{H}_{19} \mathrm{NO}+\mathrm{H}\right]^{+}\right)$. e Chemical structure of Tinuvin ${ }^{\circledR} 770$

Table 1 Main analytes detected by DART-MS and SPME-GC-MS analysis in the samples

\begin{tabular}{|c|c|c|c|c|}
\hline Sample & TMP-ol & Dimethyl sebacate & 2,4-dichlorophenol & $\begin{array}{l}\text { Chloroprene } \\
\text { derivatives }\end{array}$ \\
\hline Efflorescence & DART, SPME & & & \\
\hline Structural adhesive (white) ${ }^{*}$ & DART, SPME & SPME & SPME & SPME \\
\hline Magnetic (door) gasket & & & SPME & SPME \\
\hline Silicone (desiccant drawer) gasket & & & SPME & \\
\hline
\end{tabular}

*Terostat MS937 
On the other hand, TMP-ol and dimethyl sebacate were not identified by SPME-GC-MS in construction materials classified as magnetic (door) gaskets, silicone (desiccant drawer) gaskets, paint and silica gel beads (Table 1).

In parallel, the 26 samples of construction material sampled at NMAI-NY were analyzed by DART-MS. The samples classified as structural adhesive showed a clear ion peak $(\mathrm{m} / z$ 158.1527) associated to TMP-ol. DART-MS results match with SPME-GC-MS findings that positively identified TMP-ol in the structural adhesive (Terostat MS 937). However, while TMP-ol was not detected by SPME-GC-MS in any gasket materials, DART-MS shows the ion peak at $\mathrm{m} / \mathrm{z} 158.1527$ in two silicone (desiccant drawer) gasket samples. This could be due to: (i) the low concentration of this substance in the gaskets being undetectable by GC-MS and (ii) a crosscontamination effect, in which VOCs released during the degradation of materials can be adsorbed by nearby material during the course of the exhibition [20].

The detection of TMP-ol in the modified silicone (MS) polymer Terostat by SPME-GC-MS and DARTMS, is in agreement with previous studies from the Museum of Fine Arts (Boston, USA) and the Rijksmuseum (Amsterdam, The Netherlands) carried out in similar display cases [1-3]. These studies also linked the efflorescence with the presence of Tinuvin ${ }^{\circledR} 770$, used as an additive in the structural adhesive (Terostat) applied during the manufacture of their display cases. The preparation method of Tinuvin ${ }^{\circledR} 770$ describes the amount of substance ratio of sebacic derivative and TMP-ol as 1: (2-2.2), confirming the excess of TMP-ol in the initial mixture [5]. This excess of TMP-ol gives rise to free TMP-ol in the final composition of Terostat [3]). In the Infinity of Nations exhibition casework, the free TMPol has migrated from the structural adhesive (Terostat MS 937) to the case environment. Regardless of the low amount of HALS (concentrations range between 0.1 and $1.0 \%)$ present in the structural adhesive used within the NMAI-NY cases, the airtight cases allowed the accumulation of TMP-ol, promoting undesirable reactions and leading to efflorescence formation over time.

However, the mechanism of formation of the efflorescence is still unclear. On one hand, the molecular configuration of TMP-ol will determine its reactivity with other molecules; the nitrogen atom of the amine molecule is partially shielded by the methyl groups (Fig. 5c), preventing larger molecules from approaching easily to react with the nitrogen [21]. Therefore, the reactivity of this compound will be limited to small molecules. In addition, the reactivity of HALS has been thoroughly studied, showing that they cannot be used with halogen-containing compounds, such as PVC or halogenated materials, due to the fast generation of unwanted side reactions [22]. For example, the interaction of brominated flame retardants with alkaline hindered amines produces the deactivation of hindered amine by hydrogen bromide [23].

On the other hand, other authors have recently contemplated an acid-base reaction between TMP-ol and different acid substances. The acid donates a proton to the TMP-ol resulting into a positive ammonium cation and a negative carboxylic anion [3].

In the NMAI-NYC cases, TMP-ol seems to react with a chlorinated compound giving rise to a chlorinated salt. Although the presence of chlorine in the efflorescence has been detected by SEM-EDS, the potential source of chlorinated compounds is not clear. Previous studies have highlighted that museum artifacts may be one of the sources of chlorine [1]. The identification of a chlorinated efflorescence suggests that the crystals are associated with residues on the objects displayed at NMAI-NYC, such as chlorides in archeological ceramics, tidelines on wood and deposits such as sweat or body oil on textiles, likely from use. In addition, our analysis of construction materials by SPME-GC-MS revealed four chlorinated compounds (2,4-dichlorophenol and three chloroprene products) not originating from the objects. This illustrates that other potential sources of $\mathrm{Cl}$ should be taken into account in further research in order to elucidate the formation of the efflorescence.

2,4-dichlorophenol, detected in 13 materials (both types of gaskets and the structural adhesive Terostat) (peak 1, Fig. 6 and Table 1) has been cited among 11 priority pollutants by the US Environmental Protection Agency (EPA) list [24]. Chlorophenols are widely used as intermediates during the manufacturing of dyes, wood products, plastics, pesticides, and disinfectants [25]. A literature review shows the affinity between chlorophenols and amine compounds. Amines affected the hydrodechlorination ( $\mathrm{Cl}$ loss) reactivity or selectivity of chlorophenols [26]. This is possibly due to the alkaline and acid interactions among the amine functional groups and chlorophenols [27].

Additionally, three chloroprene products (1-chloro-5-(1-chloroethenyl)-cyclohexene, 1-chloro-4-(1-chloroethenyl)-cyclohexene, and 1,6-dichloro-1,5-cyclooctadiene) were detected in materials classified as magnetic (door) gasket and structural adhesives (peaks 2, 3 and 4, Fig. 6 and Table 1). These products have been classified as dimerization products of chloroprene (2-chloro-1,3-butadiene), a monomer used during the production of rubber materials [28]. Chloroprene can polymerize spontaneously at room temperature in a process catalyzed by light, peroxides, and other free-radical initiators, generating the three compounds 

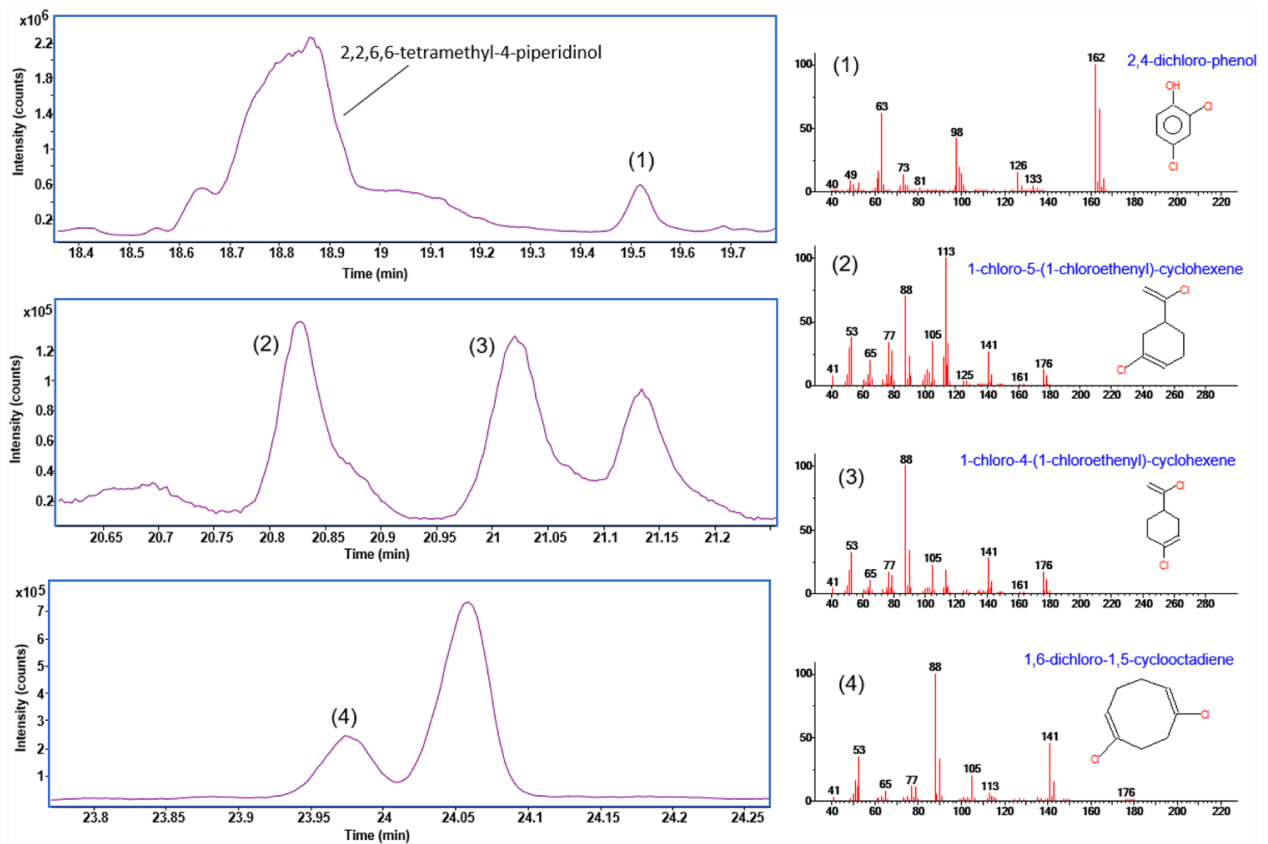

Fig. 6 Peaks in a total ion chromatogram of the structural adhesive. Corresponding mass spectra for the identified peaks associated to chlorinated compounds emitted by the structural adhesive at room temperature

identified (among others). No chloroprene products were detected in silicone (desiccant drawer) gasket samples.

Other VOCs released by the case materials such as acetic acid, benzene derivatives, oximes, aldehydes, and alcohols were also identified by SPME-GC-MS. The presence of these substances in the museum environment can initiate undesirable reactions causing damage to the collection $[7,18,29,30]$. The monitoring of these compounds will be investigated in a later phase of the project, which will focus on developing mitigation strategies to reduce the VOCs accumulated in the cases.

It is important to note that in the materials sampled at NMAAHC and CHSDM, TMP-ol was not detected by either MS technique. This finding corroborates the case manufacturer's claim that a different structural adhesive was used after 2014.

\section{On-site headspace analysis inside the museum gallery and exhibition cases.}

The detection of TMP-ol in the exhibition cases can help indicate the presence (or future formation) of the efflorescence even when it is visually hard to notice. For this reason, 32 locations including airtight showcases and open exhibition areas were analyzed over a period of 15 months by SPME-GC-MS. The selection of the locations was based on the presence or absence of visible signs of efflorescence on objects observed during previous on-site condition surveys.
SPME fibers were placed inside the cases for $24 \mathrm{~h}$. A recent publication has shown a good compromise between sensitivity and prolonged exposure times of SPME fibers, allowing the accumulation of sufficient analyte onto the fiber within a reasonable timeframe $[18,19]$. However, it should be emphasized that long exposure times can create fluctuations on the extraction yield of the analytes. The time needed to reach the equilibrium on the SPME fiber directly relies on the physicochemical parameters of each analyte; while some VOCs can take less than a few minutes, others will need a few hours to adsorb. Thus, under non-equilibrium conditions, quantitative analysis can be erroneous [31]. In addition, due to the large variation in volume between the exhibition cases (between 16-53 $\mathrm{m}^{3}$ wall cases and between $0.3-3 \mathrm{~m}^{3}$ freestanding and focal points cases) no comparison of the peak intensity was possible in this study. It should be noted that replicate sampling of this type of analysis (exhibition area or object) may provide widely variable results due to the fluctuations in the case environment and the passive nature of the SPME sampling strategy. For the above reasons, the current study focuses on qualitative interpretation of the results.

Total ion chromatograms of exhibition area, Headdresses, Andes, Eastern Woodlands and Contemporary wall cases are plotted in Fig. $7 \mathrm{a}-\mathrm{e}$ for comparison. Although no significant differences were observed between the exhibition cases, some VOCs, 
(See figure on next page.)

Fig. 7 Gas chromatogram profiles of: a background of gallery area, b Eastern Woodlands wall case, c Andes wall case, $\mathbf{d}$ Headdresses wall case and e Contemporary wall case

such as 2-butoxuethanol, 2-ethyl-1-hexanol, 2,2,4-trimethyl-1,3-pentanediol-diisobutyrate and propanoic acid-2-methyl-3-hydroxy-2,4,4-trimethylpentyl ester, were notable in the background of the gallery outside the cases (Fig. 7a). Identifying the potential sources of all VOCs detected in the exhibition cases can pose an arduous task and was not the primary goal of this study. For example, acetic acid detected in all sampling points can be either emitted by the construction materials analyzed in the previous section or by the objects $[18,31,32]$. The presence of aldehydes can be associated to the degradation of textiles or cellulosic materials [33]. The detection of naphthalene, especially in the cases containing organic objects, may be due to its historic use as pesticide during the twentieth century in many museum collections [34]. Alkylbenzene derivatives are known to be commonly present in the atmosphere and are considered indoor pollutants [18].

Notably, the detection of TMP-ol in two exhibition cases, where the efflorescence had not previously been visually identified, encouraged the conservators to look more closely for the formation of new efflorescence, finding it in both cases.

\section{On-site direct-contact SPME analysis in the exhibition cases}

To improve the detection of TMP-ol, direct-contact SPME sampling, inside the exhibition cases, was investigated to characterize the efflorescence directly on the surface of the objects on display, without the need to collect a sample (Additional file 1: Fig S4a). Results shown in Additional file 1: Fig. S4b confirm that the detection of TMP-ol is possible via the on-site direct-contact SPME sampling methodology, thus avoiding sampling the efflorescence in future research campaigns. Although direct contact SPME improved the efficiency of the detection of TMP-ol with respect to the analysis of the exhibition case (see Fig. 7d, e), the chromatogram profile showed more peaks than when the fiber was exposed solely to the efflorescence inside a vial (Fig. 5a). Indeed, other VOCs present in the exhibition case are also adsorbed onto the fiber.

\section{SPME transportation methods}

Because the exhibition (the sampling site) was located in New York and the analytical laboratory was in Maryland, the impact of transportation methods on the SPME results was studied. Two different transportation methods were evaluated: hand-carrying and commercial express mail.

Two different objects were sampled in duplicate by direct contact (Kwakwaka'wakw headdress (NMAI 23/8252) and Paviotso (Northern Paiute) duck decoy (NMAI 13/4190), Additional file 1: Fig. S5a, b). In this study, the fiber was retracted inside the needle and sealed with a pre-drilled septum. During the transportation, the temperature did not exceed the $20{ }^{\circ} \mathrm{C}$ within either transportation protocol. All fibers were analyzed with the same instrument on the same day to avoid dayto-day fluctuations. Although it has been shown that transportation under refrigeration improves the preservation of the fiber [35], transportation without refrigeration was selected based on the results of previous publication [18, 34]. A qualitative comparison of the two modes of transportation shows that the overall quality of the chromatogram obtained for the duck decoy was not affected (Additional file 1: Fig. S5c-d). Similar results were obtained for the headdress. We have demonstrated here that the SPME fibers can be easily exposed in the exhibition by the conservator or collections manger. In addition, the third party shipping method poses a good alternative for those museums without access to advanced analytical instrumentation.

\section{Conclusions and future perspectives}

In this study, two mass spectrometry approaches, SPME-GC-MS and DART-MS, were used to investigate the efflorescence detected in a quarter of the objects exhibited in Infinity of Nations at the NMAI-NY. This is the first time these techniques have been used for the analysis of efflorescence formed on historically and culturally valuable objects. Both techniques showed great potential for the analysis of: (1) the efflorescence, (2) the exhibition cases materials, and (3) VOCs accumulated in display cases that could lead to the formation of the efflorescence.

Both SPME-GC-MS and DART-MS identified TMPol as the (organic) component of the efflorescence. MS methods linked the presence of TMP-ol to the structural adhesive (Terostat MS 937) used for construction of the exhibition cases. Additionally, SPME-GC-MS provided a qualitative identification of the various chlorinated VOCs, released by the exhibition case materials at room temperature, that can lead to formation of the efflorescence. 

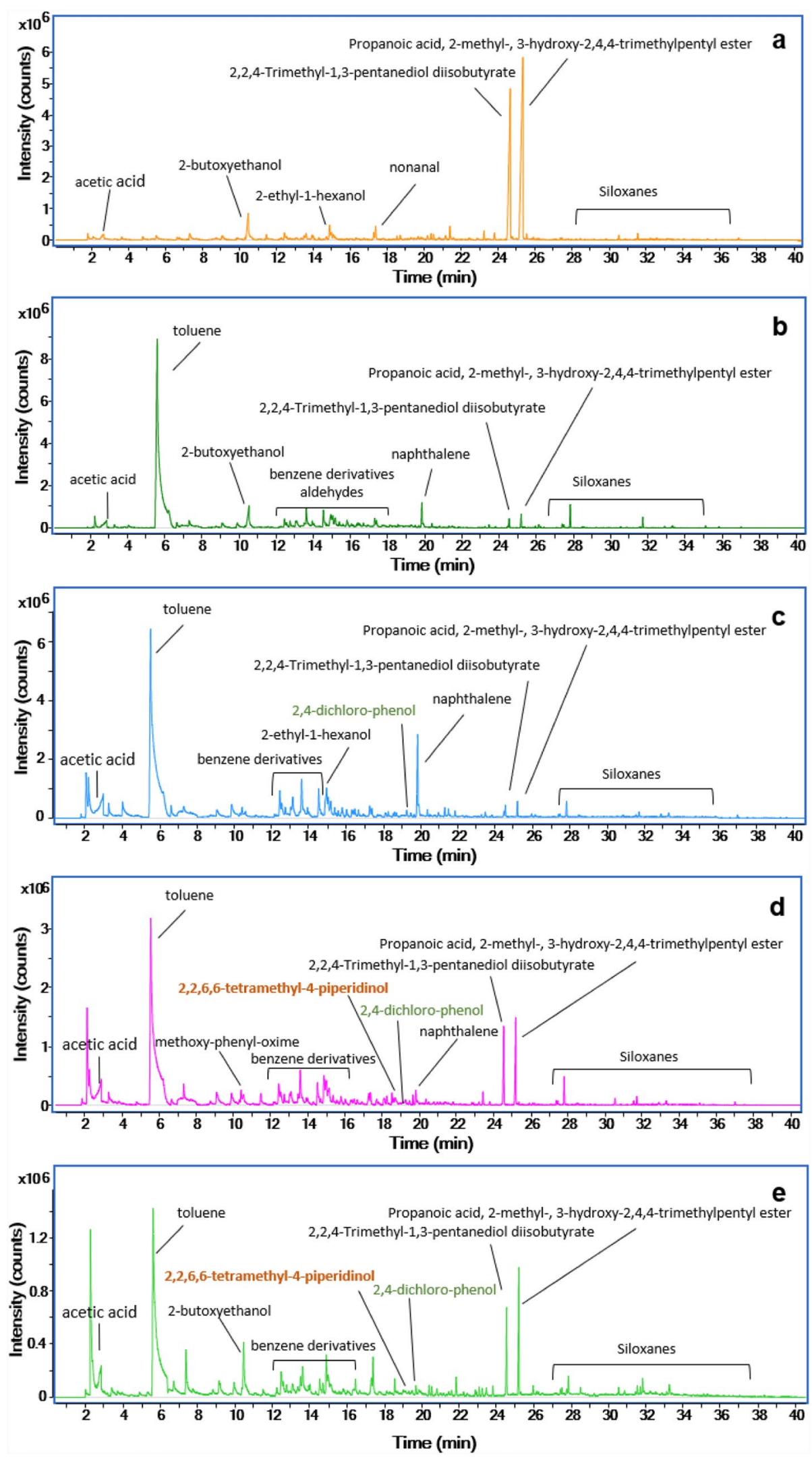
While several hours of operating time are needed for SPME, including sampling, thermal desorption in the GC, chromatographic separation, MS detection, and data analysis, DART-MS drastically reduced the time for analysis. Incorporating DART-MS as a routine method for material screening could greatly reduce the time for analysis, from the 28 days for the Oddy test or two or three hours for SPME-GC-MS to only a few minutes for DART-MS. However, DART does not have a very large spectrum database or software platform as well-developed and/or commonly used as the NIST EI mass spectrum database and matching algorithm, when compared to GC-MS. Nevertheless, the combination of both techniques provides a full understanding of the main VOCs released by the materials (via GC-MS), followed by a fast detection of TMP-ol (via DART-MS).

The main drawback of the application of MS techniques in cultural heritage projects is that these techniques require access to advanced laboratory facilities. In order to broaden the application of MS to museums with limited resources, the potential of SPME as a portable and easy-to-operate sampling technique in museums is shown in this study. The SPME sampling protocol proposed here allows conservators to receive and expose the SPME fibers in the display case without the presence of a conservation scientist. After sampling, the fibers can be shipped overnight to the laboratory for analysis on the following day.

However, in the current study we were not able to give concentration levels of TMP-ol. A quantitative analytical protocol will be developed in the next phase of this project to determine the levels of TMP-ol at which the crystallization occurs in the exhibition cases. The research presented here is the preliminary phase of a large scientific and conservation project. The rapid identification of the source of the efflorescence and its volatile compound by SPME-GC-MS and DART-MS allowed to better understand the phenomena occurring in the Infinity of Nations display cases. The outcome of this investigation will culminate in the development of a robust protocol for the rapid detection of volatiles from case interiors and a method for measuring the efficacy of mitigation strategies used to reduce the VOCs accumulated in the display cases. In addition, these MS methods can be implemented during the selection process for new materials to limit future unwanted reactions in the display cases.

\section{Supplementary information}

Supplementary information accompanies this paper at https://doi. org/10.1186/s40494-020-00454-4.

Additional file 1: Table S1. Efflorescence sampled at NMAI-NY. Table S2. Construction materials sampled at NMAI-NY. Table S3. Exhibition cases analysed by SPME-GC-MS. Figure S1. DART-MS configuration for the analysis of: a exhibition case materials and $\mathbf{b}$ efflorescence. Figure $\mathbf{S 2}$. Diffraction patterns of the efflorescence formed on a Chican Ostionoid wooden bowl; 04/2409 and b Kwakwaka'wakw headdress cotton fabric; 23/8252. Figure S3. ATR-FTIR spectrum of Chican Ostionoid wooden bowl; 04/2409. Figure S4. a SPME fiber in direct contact with Manteño-Huancavilca stone seat; 01/6380 and (b) GC-MS Total Ion Chromatogram (TIC) profile of the same fiber. Figure S5. Duplicate SPME fibers in direct contact with: a Kwakwaka'wakw headdress; 23/8252 and b Paviotso (Northern Paiute) duck decoy; 13/4190. GC-MS Total Ion Chromatogram (TIC) profile of duck decoy transported by: $\mathbf{c}$ express mail and $\mathbf{d}$ hand-carry.

\section{Abbreviations}

NMAI-NY: National Museum of the American Indian-New York; SPME: Solid phase microextraction; GC: Gas Chromatography; DART: Direct Analysis Real Time; MS: Mass Spectrometry; SEM-EDS: Scanning Electron MicroscopyEnergy Dispersive X-ray Spectroscopy; XRPD: X-Ray Powder Diffraction; ATRFTIR: Attenuated total reflection- Fourier transform infrared spectroscopy.

\section{Acknowledgements}

AAM acknowledges the Smithsonian Postdoctoral Fellowship in the Conservation of Museum Collections and the Museum Conservation Institute. LB and LO acknowledge the Andrew W. Mellon Foundation for their support of Fellowships in Conservation at the National Museum of the American Indian. The authors thank Gerry Breen and Sarah Elston (NMAI-NY) for providing the elevations and floor plans and Dr. Thomas Lam (MCI) for SEM-EDS analysis.

The authors sincerely acknowledge Richard Newman and Pamela Hatchfield (MFA, Boston) for providing valuable information over the years.

\section{Authors' contributions}

AAM, SH, LO, JG, EK, RK and LB selected the sampling locations and performed the sampling. AAM performed SPME-GC-MS analysis. AAM and GAN performed DART-MS research. GK performed the ATR-FTIR research. FV performed the XRPD analysis. AAM wrote the manuscript, with edits and comments from all other participants. All authors read and approved the final manuscript.

\section{Funding}

This project received U.S. Federal support from the Collections Care Initiative administered by the Smithsonian National Collections Program. The project was also supported by the Smithsonian's National Museum of the American Indian and the Museum Conservation Institute.

\section{Availability of data and materials}

All data generated or analyzed during this study are included in this published article. More information is available from the corresponding author on reasonable request.

\section{Competing interests}

The authors declare that they have no competing interests.

\section{Author details}

${ }^{1}$ Museum Conservation Institute, Smithsonian Institution, 4210 Silver Hill Road, Suitland, MD, USA. ${ }^{2}$ The National Museum of the American Indian, Smithsonian Institution, 1 Bowling Green, New York, NY, USA. ${ }^{3}$ Cultural Resources Center, The National Museum of the American Indian, Smithsonian Institution, 4220 Silver Hill Road, Suitland, MD, USA. ${ }^{4}$ Department of Physics, AXES, 171 Groenenborgerlaan, Antwerp, Belgium. ${ }^{5}$ Present Address: Conservation \& Science, Rijksmuseum, Hobbemastraat 22, 1071 ZC Amsterdam, The Netherlands.

Received: 30 May 2020 Accepted: 26 October 2020

Published online: 14 November 2020

\section{References}

1. Newman R, Derrick M, Byrne E, Tan M. Strange events inside display cases at the museum of fine arts, boston, and lessons to be learned from them-part 1 conservation and exhibition planning: material testing 
for design, display, and packing. Washington: Smithsonian American Art Museum \& National Portrait Gallery ; 2015.

2. Hatchfield P, Goppion S, Chiantore O, Poli T, Riedo C, Suslick K, et al. Strange events inside display cases at the museum of fine arts, boston, and lessons to be learned from them - part 2 conservation and exhibition planning: material testing for design, display, and packing. Washington: Smithsonian American Art Museum \& National Portrait Gallery; 2015.

3. van Iperen J, van Keulen H, Keune K, Abdulah K, van Langh R. Crystalline deposits in new display cases at the rijksmuseum: characterisation andorigin. Stud. Conserv. 2020. https://doi.org/10.1080/00393630.2020.18114 75 .

4. US4396769A. Process for preparing crystalline, non-dusting bis(2,2,6,6tetramethylpiperidin-4-yl) sebacate. United States 1982. https://paten ts.google.com/patent/US4396769A/en.

5. CN103980185A. Preparation method of hindered amine light stabilizer 770. China 2014. https://patents.google.com/patent/CN103980185A/en.

6. Shikh Zadeh M, Rabiei R, Sheibani R. The Study of the Possibility of Using Expired Ultraviolet Stabilizer (Tinuvin 770) in Polypropylene. Eur Online J Nat Soc Sci. 2016;5:484-93.

7. Oddy WA. An unsuspected danger in display. Museum J. 1973;73:27-8.

8. Tsukada M, Rizzo A, Granzotto C. A new strategy for assessing off-gassing from museum materials: air sampling in oddy test vessels. Am Inst Conserv Historic Artistic Works. 2012;37:1-7.

9. Samide MJ, Liggett MC, Mill J, Smith GD. Relating volatiles analysis by GCMS to Oddy test performance for determining the suitability of museum construction materials. Herit Sci. 2018;6(1):47.

10. Samide MJ, Smith GD. Analysis and quantitation of volatile organic compounds emitted from plastics used in museum construction by evolved gas analysis-gas chromatography-mass spectrometry. J Chromatogr A. 2015;1426(Supplement C):201-8.

11. Stephens $\mathrm{CH}$, Buscarino I, Breitung E. Updating the oddy test: comparison with volatiles identified using chromatographic techniques. Stud Conserv. 2018;63(sup 1):425-7.

12. Schieweck A, Salthammer T. Emissions from construction and decoration materials for museum showcases. Stud Conserv. 2009;54(4):218-35.

13. Chiantore O, Riedo C, Poli T, Cotrufo G, Hohenstatt P. Risk assessment and preservative measures for volatile organic compounds in museum showcases. Stud Conserv. 2018;63(sup1):58-63.

14. US5691206A. Method and device for solid phase microextraction and desorption. Canada 1990. https://patents.google.com/patent/US569 1206A/en.

15. Newsome GA, Kavich G, Alvarez-Martin A. Interface for reproducible, multishot direct analysis of solid-phase microextraction samples. Anal Chem. 2020;92(6):4182-6.

16. Gross JH. Direct analysis in real time-a critical review on DART-MS. Anal Bioanal Chem. 2014;406(1):63-80.

17. Cody RB, Fouquet TNJ, Takei C. Thermal desorption and pyrolysis direct analysis in real time mass spectrometry for qualitative characterization of polymers and polymer additives. Rapid Commun Mass Spectrom. 2020;34(S2):e8687.

18. Alvarez-Martin A, McHugh K, Martin C, Kavich G, Kaczkowski R. Understanding air-tight case environments at the National Museum of the American Indian (Smithsonian Institution) by SPME-GC-MS analysis. J Cult Herit. 2020. https://doi.org/10.1016/j.culher.2020.01.004.

19. Kearney M, Parkin I, Townsend JH, Hidalgo M, Curran K. Characterisation of VOCs surrounding Naum Gabo's construction in space 'Two
Cones', (Tate) by in situ SPME GC-MS monitoring. Stud Conserv. 2018;63(sup1):369-71.

20. Curran K, Možir A, Underhill M, Gibson LT, Fearn T, Strlič M. Crossinfection effect of polymers of historic and heritage significance on the degradation of a cellulose reference test material. Polym Degrad Stab. 2014;107(Supplement C):294-306

21. Mokhatab S, Poe WA, John M. Condensate Production. In: Mokhatab S, Poe WA, Mak JY, editors. Handbook of natural gas transmission and processing, 4th ed. Gulf Professional Publishing; 2019. p. 219-29.

22. Kutz M. Applied plastics engineering handbook, 2nd ed. William Andrew; 2016.

23. Wypych G. Handbook of UV degradation and stabilization, 2nd ed. ChemTec Publishing; 2015. p. 67-139.

24. United States Environmental Protection Agency. https://www.epa.gov/ toxics-release-inventory-tri-program/list-tri-chemicals-within-chlorophen ols-category. Accessed May 2020

25. Ho T-T, Chen C-Y, Li Z-G, Yang TC-C, Lee M-R. Determination of chlorophenols in landfill leachate using headspace sampling with ionic liquid-coated solid-phase microextraction fibers combined with gas chromatography-mass spectrometry. Anal Chim Acta. 2012;712:72-7.

26. Ma X, Zhou S, Yang C, Liu S, Bi X, Xia C. The influence of triethylamine on the hydrodechlorination reactivity of chlorophenols over Raney $\mathrm{Ni}$ catalyst. Catal Commun. 2010;12(4):282-5.

27. Ghaffari A, Tehrani MS, Husain SW, Anbia M, Azar PA. Adsorption of chlorophenols from aqueous solution over amino-modified ordered nanoporous silica materials. J Nanostruct Chem. 2014;4(3):114.

28. Kwak J, Fan M, Martin JA, Ott DK, Grigsby CC. Dimerization products of chloroprene are background contaminants emitted from ALTEF (Polyvinylidene Difluoride) gas sampling bags. Anal Sci. 2017;33(2):147-52.

29. Curran K, Strlič M. Polymers and volatiles: Using VOC analysis for the conservation of plastic and rubber objects. Stud Conserv. 2015:60(1):1-14.

30. Hatchfield PB. Pollutants in the museum environment. London: Archetype Publications Ltd.; 2002.

31. Lattuati-Derieux A, Thao S, Langlois J, Regert M. First results on headspace-solid phase microextraction-gas chromatography/mass spectrometry of volatile organic compounds emitted by wax objects in museums. J Chromatogr A. 2008;1187(1):239-49.

32. Ryhl-Svendsen M, Glastrup J. Acetic acid and formic acid concentrations in the museum environment measured by SPME-GC/MS. Atmos Environ. 2002;36(24):3909-16.

33. Zhu H, Lu Z, Cai J, Li J, Gao L. Development of a headspace-SPME-GC/MS method to determine volatile organic compounds released from textiles. Polym Test. 2009;28(5):521-7.

34. Ormsby M, Johnson JS, Heald S, Chang L, Bosworth J. Investigation of solid phase microextraction sampling for organic pesticide residues on museum collections. Collection Forum. 2006;20(1-2):1-12.

35. Koziel J, Jia M, Khaled A, Noah J, Pawliszyn J. Field air analysis with SPME device. Anal Chim Acta. 1999;400(1):153-62.

\section{Publisher's Note}

Springer Nature remains neutral with regard to jurisdictional claims in published maps and institutional affiliations. 